\title{
ARTICLE \\ Inhibition of the rostromedial tegmental nucleus reverses alcohol withdrawal-induced anxiety-like behavior
}

\author{
Elizabeth J. Glover $\mathbb{D}^{1,2}$, E. Margaret Starr ${ }^{1,2}$, Ying Chao ${ }^{1}$, Thomas C. Jhou ${ }^{1}$ and L. Judson Chandler ${ }^{1,2}$
}

\begin{abstract}
Acute withdrawal from alcohol is associated with a number of unpleasant symptoms that play an important role in preventing recovery and long-term abstinence. Considerable research has focused on the role that neuropeptide systems and the amygdala play in mediating affective symptoms of acute withdrawal, but promising preclinical findings have not translated successfully into the clinic. The rostromedial tegmental nucleus (RMTg) has been implicated in both fear and anxiety. In addition, RMTg neurons exert inhibitory control over midbrain dopamine neurons, the activity of which are suppressed during acute withdrawal. Thus, we hypothesized that the RMTg may play a role in mediating symptoms of acute withdrawal. Using a chronic ethanol vapor exposure paradigm that renders rats physically dependent on ethanol, we observed significant withdrawal-induced enhancement of cFos expression in the RMTg. This was accompanied by a significant increase in somatic symptoms and a decrease in reward sensitivity as measured by intracranial self-stimulation (ICSS). Both measures followed a similar time course to RMTg cFos expression with peak symptom severity occurring $12 \mathrm{~h}$ following cessation of ethanol exposure. Heightened anxiety-like behavior was also observed in withdrawn rats at this same time point. RMTg inhibition had no effect on somatic signs of withdrawal or withdrawal-induced changes in reward sensitivity, but significantly attenuated withdrawal-induced anxiety-like behavior. Together, these data demonstrate that the RMTg plays a distinct role in the negative affective state associated with acute withdrawal and may therefore be critically involved in the neurobiological mechanisms that promote relapse during early stages of recovery.
\end{abstract}

Neuropsychopharmacology (2019) 44:1896-1905; https://doi.org/10.1038/s41386-019-0406-8

\section{INTRODUCTION}

Acute withdrawal from alcohol is among the criteria for an alcohol use disorder (AUD) and results in a constellation of somatic and affective symptoms [1]. Alcohol-dependent individuals are frequently motivated via negative reinforcement to drink to alleviate these symptoms [1, 2]. Rodent models of dependence have effectively recapitulated both somatic and affective withdrawal symptoms. For example, chronic ethanol exposure is associated with signs of rigidity and motor impairment [3-8], increased anxiety-like behavior $[3,9,10]$, and decreased reward sensitivity [7]. In agreement with negative reinforcement driving alcohol consumption, a number of studies have shown that animals self-administer ethanol in greater quantities during acute withdrawal compared with pre-dependence levels [11-14] and that ethanol intake at this time prevents the development of withdrawal symptoms [6]. Moreover, animals allowed to drink during acute withdrawal exhibited higher levels of intake relative to those whose intake was confined to protracted withdrawal once acute symptoms have subsided [15]. These data suggest that a critical window exists during the first $24 \mathrm{~h}$ of withdrawal whereby the inability to remain abstinent can be detrimental to long-term efforts to curb drinking.

Like withdrawal from other drugs of abuse, alcohol withdrawal is associated with reduced activity in ventral tegmental area (VTA) dopamine neurons [8]. This is accompanied by decreased extracellular dopamine levels in the nucleus accumbens that, similar to symptoms of withdrawal, is greatest $8-12 \mathrm{~h}$ following cessation of ethanol exposure $[8,16,17]$ and is reversed upon re-exposure to ethanol [8, 17]. Despite the correlation between VTA dopamine release and withdrawal symptoms, considerable research aimed at understanding the neurobiology of withdrawal-induced negative affect has focused on neuropeptide systems like corticotropin-releasing factor (CRF) and the amygdala due to their overlapping involvement in both AUDs and anxiety [18]. Although manipulation of the CRF system has shown promise in reducing withdrawal symptoms in animal models $[3,10,19,20]$, recent clinical trials produced negative findings [21, 22]. While by no means conclusive ([23]; see ref. [24]), these observations underscore the importance of exploring alternative neurobiological substrates that may play a role in withdrawal and therefore pose new targets to aid in recovery from AUDs.

One such potential target is a small GABAergic region known as the rostromedial tegmental nucleus (RMTg), also known as the tail of the VTA (tVTA). First identified in 2009, the RMTg receives glutamatergic input from the lateral habenula (LHb), sends a dense projection to the VTA thereby exerting inhibitory control over midbrain dopamine neurons, and is delineated by expression of the transcription factor FoxP1 [25-27]. This neural circuit (LHb-RMTg-VTA), which exhibits only modest direct connectivity

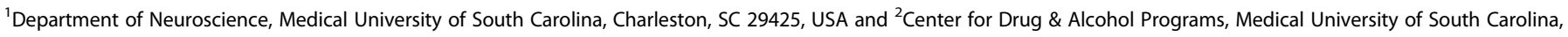
Charleston, SC 29425, USA

Correspondence: Elizabeth J. Glover (ejglover@uic.edu)

Joint senior authors: Thomas C. Jhou, L. Judson Chandler

Received: 18 November 2018 Revised: 24 April 2019 Accepted: 26 April 2019

Published online: 6 May 2019 
with the amygdala [28], has been characterized for its involvement in negative reward prediction error [29]. In addition, similar to the amygdala, the RMTg plays a role in both fear expression and anxiety-like behavior [30], as well as conditioned avoidance in response to acute withdrawal from cocaine [31] and punishment [32]. Given its functional relevance and role in regulating dopamine release, we hypothesized that the RMTg may be involved in mediating withdrawal-induced negative affect. We tested this hypothesis in a rat model of alcohol dependence by examining the effect of RMTg inhibition on changes in reward sensitivity and anxiety-like behavior during acute withdrawal.

\section{MATERIALS AND METHODS}

Animals

Adult male Long-Evans rats (Envigo, Indianapolis, IN) were used for all experiments. All procedures were conducted with the approval of the Medical University of South Carolina's Institutional Animal Care and Use Committee, and adhered to the guidelines provided by the National Research Council's Guide for the Care and Use of Laboratory Animals [33].

\section{Chronic intermittent ethanol exposure}

Rats were rendered physically dependent upon alcohol using a chronic intermittent ethanol (CIE) vapor exposure paradigm as previously described [35]. Rats were exposed to ethanol vapor for $14 \mathrm{~h}$ per day for 14 consecutive days before initiation of studies examining the effects of withdrawal. Controls were treated similarly, except they were exposed to room air (AIR). Blood samples were obtained via tail vein puncture immediately upon removal from the vapor chambers at the end of the 2nd, 6th, 10th, and 14th exposure days. Samples were analyzed for blood ethanol concentration (BEC) using a colorimetric enzymatic assay [34] or Analox alcohol analyzer (Analox Instruments, Atlanta, GA).

To avoid chronic blood sampling, rats were also scored for behavioral signs of intoxication daily, at the end of each ethanol exposure period, using a 5-point scale as described previously [35]. Target levels of intoxication were set at $250 \mathrm{mg} / \mathrm{dL}$, which equates to $\sim 2.5$ on the 5-point behavioral intoxication scale. Because of the potential confounding effect that sampling could have on measures of withdrawal-induced negative affect, blood samples were not obtained immediately prior to withdrawal testing to demonstrate absence of intoxication. However, previous work in rats that achieved similar levels of intoxication has shown that blood ethanol levels approach zero $\sim 8 \mathrm{~h}$ after cessation of ethanol exposure with brain ethanol levels declining even more rapidly [11]. Importantly, behavioral testing was rescheduled for rats that received a score of 4 or 5 on the intoxication rating scale upon removal from the vapor chambers. Together, with our own data showing the presence of somatic signs of withdrawal observed immediately prior to testing, these data suggest that intoxication was not a confounding factor influencing behavioral performance in the experiments described below.

Intracranial self-stimulation

Intracranial self-stimulation (ICSS) was conducted in standard operant boxes (Med Associates, Fairfax, VT). Procedures were adapted from previously published methods [36]. See Supplement for additional details.

\section{Anxiety battery}

Rats were assessed for anxiety-like behavior using a battery of tests that included elevated plus maze, light-dark box, and open field. See Supplement for additional details.

Intra-RMTg infusions

Methods for muscimol microinfusions into the RMTg were adapted from previously published work [37, 38]. To assess muscimol spread, a small group of rats $(n=4)$ were cannulated and sacrificed 10 min after microinfusion of muscimol $(300 \mathrm{~nL}$, $800 \mu \mathrm{M}, \mathrm{BODIPY}$, ThermoFisher, Waltham, MA) into the RMTg at a rate of $200 \mathrm{~nL} / \mathrm{min}$. In all four rats, muscimol spread was restricted to an area within the boundaries of the RMTg (Figure S5). In agreement with previous work [37], fluorescent imaging revealed that spread was restricted medio-laterally to a diameter of $\sim 500$ $\mu \mathrm{m}$ and extended rostro-caudally to a diameter of $\sim 600 \mu \mathrm{m}$ (Fig. S5). Because BODIPY-conjugated muscimol has a molecular weight roughly fivefold higher than unconjugated muscimol, and therefore is likely to diffuse more slowly, we ensured similar levels of spread by performing all subsequent behavioral experiments using the same methods. Rats were habituated to the microinfusion procedure for 3 days prior to first infusion and re-habituated for 1 day prior to subsequent infusions. On the day of microinfusion, the dummy cannula was removed and an infusion cannula extending $1 \mathrm{~mm}$ beyond the guide was inserted and muscimol $(300 \mathrm{~nL}, 800 \mu \mathrm{M}, \mathrm{BODIPY})$ or saline $(300 \mathrm{~nL})$ was infused into the RMTg. The infusion cannula was left in place for two additional minutes before removal. Testing began 5-10 min following completion of the infusion procedure.

Statistical analysis

All statistical tests and graphs were generated using IBM SPSS Statistics Version 24 and Prism 7 Mac OS X. ICSS data were normalized to percent maximum baseline responding to enable group comparisons. Intoxication levels, histological, and behavioral data were analyzed using student's $t$ test, two-way, and multifactorial analysis of variance (ANOVA) and linear regression where applicable. Data are presented as mean \pm SEM and effects were considered statistically significant at $p \leq 0.05$.

\section{RESULTS}

Enhanced RMTg cFos expression during acute withdrawal Acute withdrawal is associated with a sequela of aversive symptoms and a significant decrease in VTA dopamine neuron activity. We therefore hypothesized that RMTg neurons would be hyperactive during this same time. To examine this, we quantified withdrawal-induced cFos expression, a marker of neuronal activity, in the RMTg and the lateral habenula ( $\mathrm{LHb}$ - - a region that sends dense input to the RMTg. As shown in Fig. 1a-d, acute withdrawal produced a significant increase in cFos expression in both regions. In the RMTg, a two-way ANOVA found significant main effects of ethanol exposure and time, and a significant interaction between factors $[\mathrm{F}(3,31)=8.468, p=0.0003]$. Post-hoc comparisons revealed that, relative to controls, cFos expression was significantly enhanced in the RMTg of CIE-exposed rats at the 6 - and 12 -h time points $(p<0.05)$ with peak cFos induction occurring at $12 \mathrm{~h}$. In the LHb, a two-way ANOVA uncovered significant main effects of ethanol exposure $[F(1,32)=16.55, p=$ $0.0003]$ and time $[F(3,32)=2.943, p=0.0478]$. However, an interaction between the two factors did not reach statistical significance $[F(3,32)=2.813, p=0.0550]$. Nevertheless, cFos induction in the LHb followed a pattern similar to the RMTg with peak expression occurring at $12 \mathrm{~h}$. cFos expression was positively correlated between regions $\left(p<0.0001, r^{2}=0.7936\right)$ consistent with previous work showing similar patterns of neuronal activation to aversive stimuli in LHb and RMTg [29]. Notably, no effect of ethanol exposure, time, or interaction between factors was observed in the medial habenula ( $\mathrm{MHb}$; Fig. $1 \mathrm{~d}$, e), a region located immediately adjacent to the LHb (all $p$-values $>0.20$ ). Furthermore, RMTg cFos expression was not significantly correlated with $\mathrm{MHb}$ cFos expression $\left(p=0.6581, r^{2}=0.0052\right)$. Importantly, withdrawal-induced cFos expression was not due to differences in level of intoxication between groups (Fig. S1). These data suggest that the RMTg is hyperactive during acute withdrawal in a pattern that correlates with previous reports of peak 


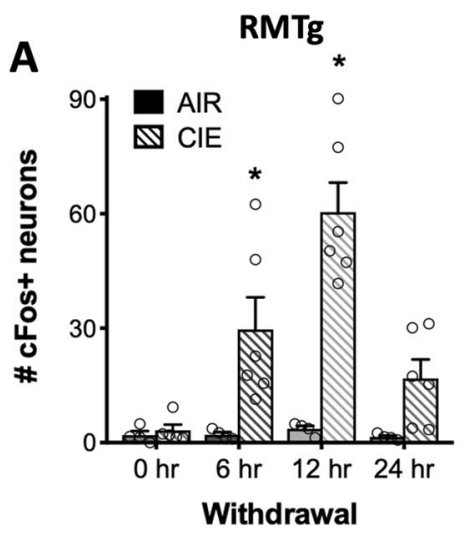

B
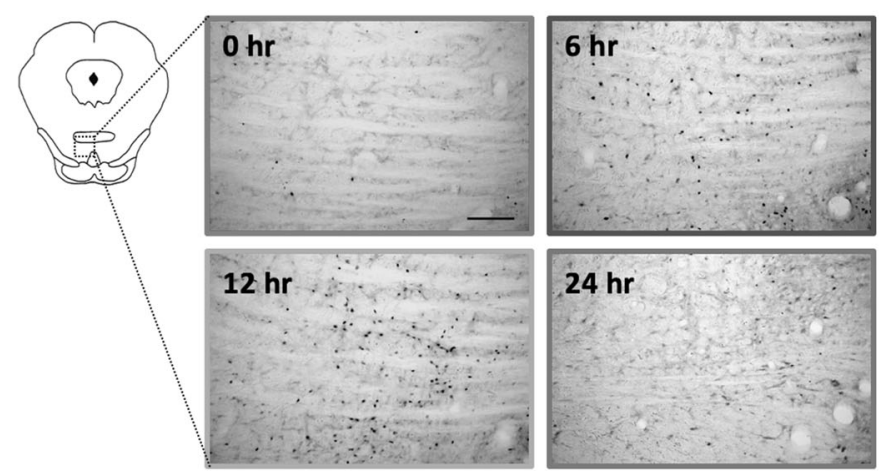

LHb
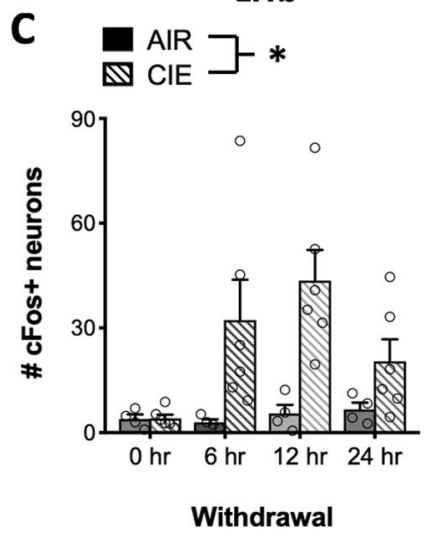

$\mathbf{E}$

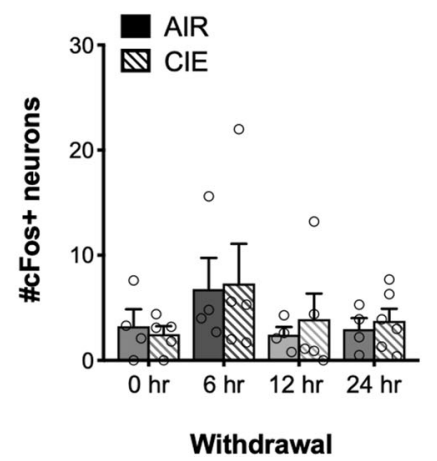

$\mathbf{F}$

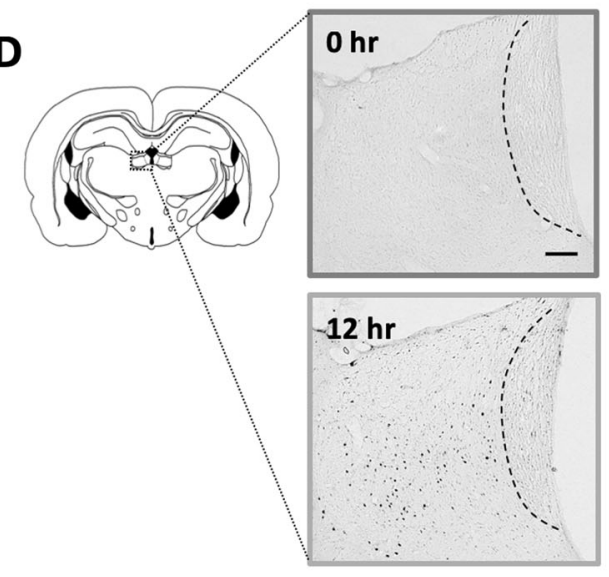

G
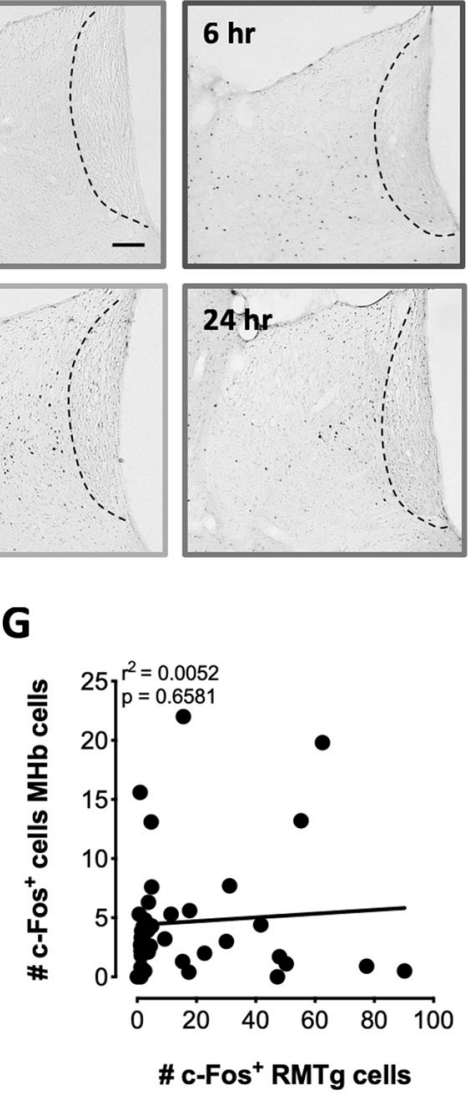

Fig. 1 Withdrawal-induced RMTg cFos. Expression of cFos was enhanced in the RMTg (a, b) and LHb (c, d) across the time course of acute withdrawal relative to controls with peak induction occurring $12 \mathrm{~h}$ following removal from the vapor chambers. In contrast, acute withdrawal from alcohol had no effect on cFos expression in the $\mathrm{MHb}(\mathbf{d}, \mathbf{e})$. Note, the boundary between MHb and LHb is demarcated by a dotted line in d. RMTg cFos expression was positively correlated with LHb cFos expression (f), but did not correlate significantly with MHb cFos expression (g). ${ }^{*} p \leq 0.05$; AIR, $n=4 ; \mathrm{CIE}, n=5-6$; scale bars $=100 \mu \mathrm{m}$

symptoms. Based on these findings, we explored the possibility that RMTg inhibition reverses symptoms of withdrawal.

Rightward shift in frequency-rate responding during acute withdrawal

ICSS was used to investigate the role of the RMTg in withdrawalinduced alterations in reward sensitivity. Figure $2 \mathrm{a}$ provides a schematic depiction of the experimental design for these studies. Of the 23 rats implanted with a stimulating electrode, six were removed from the experiment during training due to inability to meet response criteria. Figure $2 \mathrm{~b}$ shows electrode-termination sites for all remaining rats. Intoxication levels achieved during ethanol vapor exposure were similar to that which was observed in the cFos experiment (Fig. S3). Baseline frequency-rate (F-R) curves were acquired for all rats prior to being subjected to air or ethanol vapor. Rats assigned to AIR and CIE groups exhibited similar response rates at each frequency tested, and no significant between group differences were observed in the total or maximum number of responses made in a given session (Fig. S2).

After obtaining baseline responding, rats were subjected to 14 days of AIR or CIE exposure to induce physical dependence, after which time ICSS testing occurred 6, 12, and $24 \mathrm{~h}$ after removal from the vapor chamber. Rats were scored for somatic signs of withdrawal immediately prior to each ICSS session. A twoway RM ANOVA revealed a significant interaction between ethanol exposure and time $[\mathrm{F}(2,30)=4.229, p=0.0241]$. Post-hoc analyses 
A

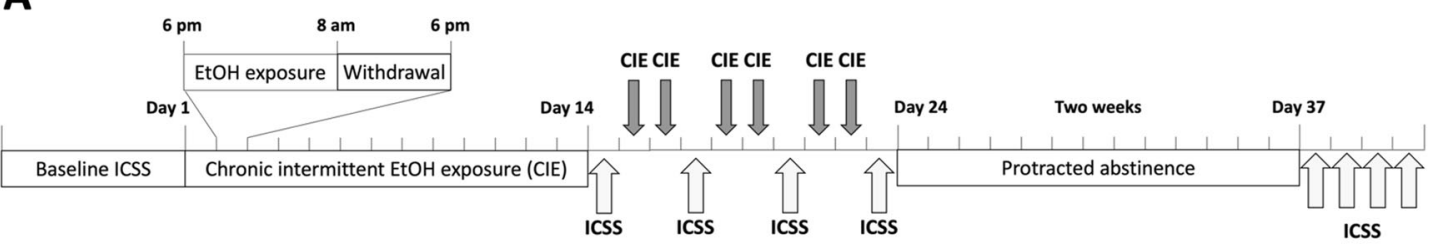

B
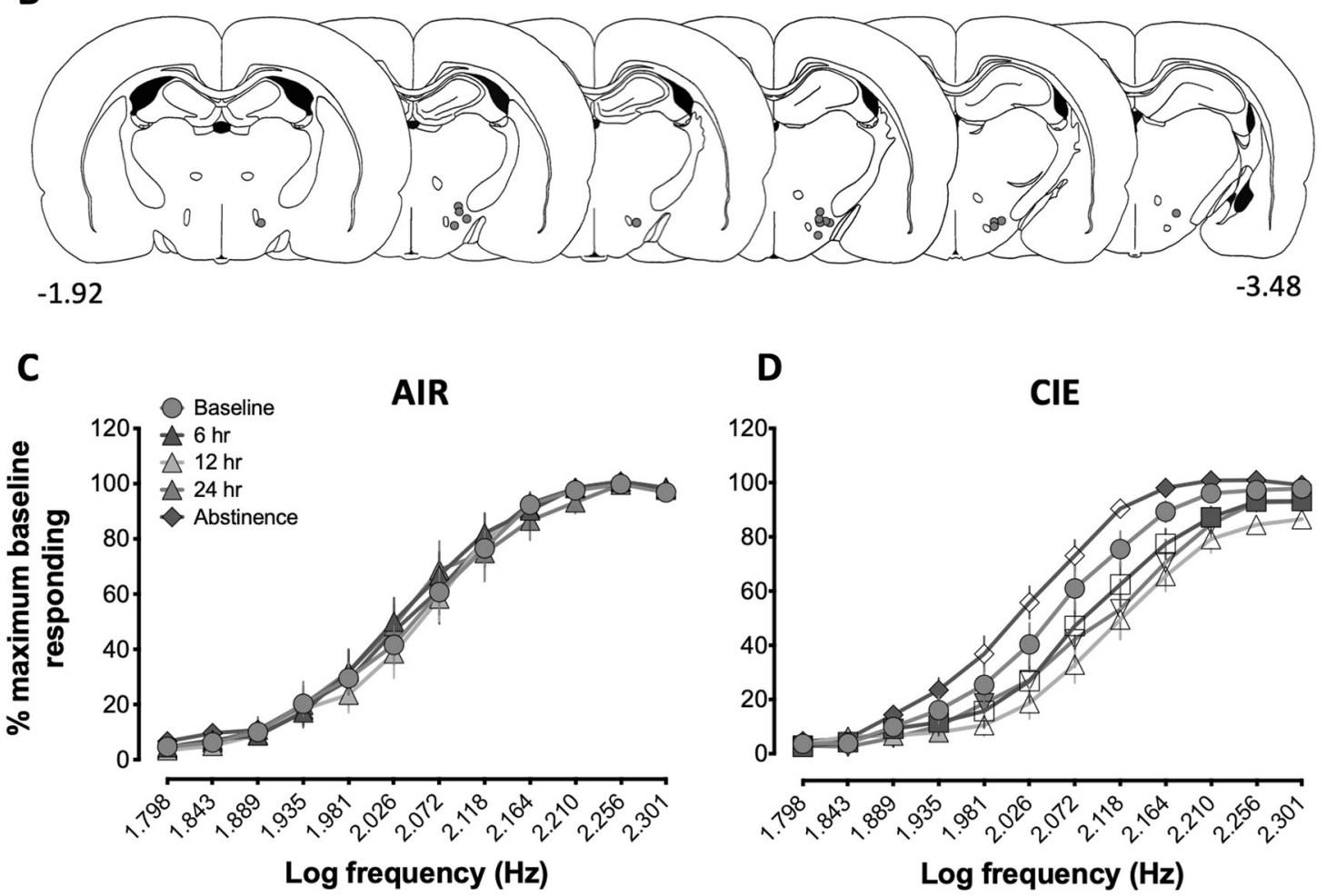

D

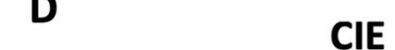

E

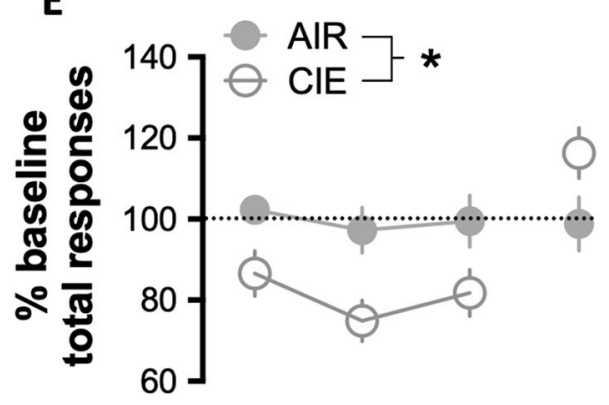

$\left.\begin{array}{r}120 \\ 100 \\ 80 \\ 60 \\ 40 \\ 20 \\ 0\end{array}\right]$

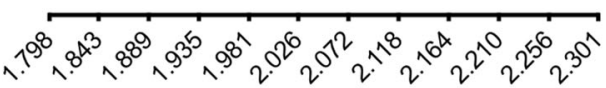

Log frequency $(\mathrm{Hz})$

$\mathbf{F}$

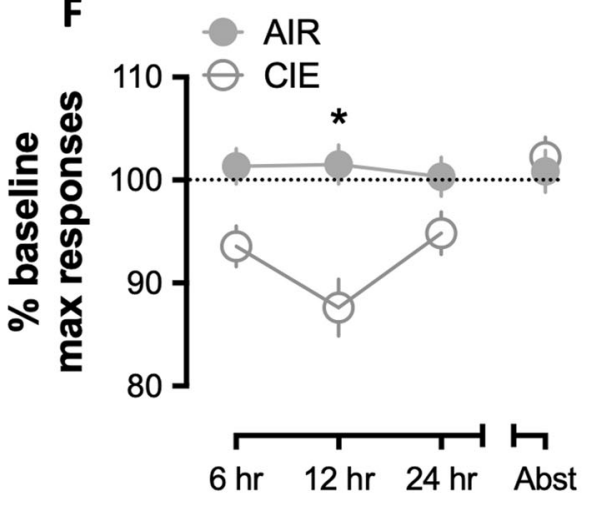

Fig. 2 Dependence-induced alterations in reward sensitivity. a Schematic depiction of experimental design and $\mathbf{b}$ electrode termination sites. c F-R responding remained stable across time in AIR-exposed rats. In contrast, CIE-exposed rats (d) exhibited a significant rightward shift in F- $\mathrm{R}$ responding across acute withdrawal with peak shift occurring at the 12-h time point. During abstinence, CIE-exposed rats exhibited a slight but significant leftward shift in F-R responding (open symbols indicate statistical significance from corresponding baseline value; $p \leq 0.05$ ). The rightward shift in F-R responding in CIE-exposed rats was associated with a significant decrease in both total responses (i.e., the sum of all responses across the F-R curve) (e) and maximum responses (i.e., the maximum number of responses within a 50-s response period) (f) made during the withdrawal session. ${ }^{*} p \leq 0.05$. AIR, $n=7 ; \mathrm{CIE}, n=10$

revealed that, as expected, CIE-exposed rats achieved significantly higher withdrawal scores than AIR-exposed rats at all time points $(p<0.0001$; Fig. S4). Similar to RMTg cFos induction, somatic signs of withdrawal peaked $12 \mathrm{~h}$ after removal from the vapor chambers (Fig. S4).
As shown in Fig. 2c, d, acute withdrawal produced a significant rightward shift in F-R responding. A three-way mixed ANOVA uncovered a significant interaction between time, frequency, and group $[F(64,896)=2.971, p<0.0005]$. Subsequent assessment of simple two-way interactions revealed a significant interaction 
between time and frequency in CIE-exposed rats $[\mathrm{F}(64,512)=$ $4.509, p<0.0005]$ that was absent in AIR-exposed rats $[\mathrm{F}(64,384)=$ $0.785, p=0.881]$. Post-hoc analysis showed that responding was significantly decreased in CIE-exposed rats across acute withdrawal. Once again, the greatest magnitude effect occurred at the 12-h time point. In contrast, during protracted abstinence, CIE-exposed rats exhibited a slight but significant leftward shift in $F-R$ responding, whereas responding was unchanged in AIRexposed rats. Alterations in F-R responding were accompanied by similar changes in the total and maximum responses. Two-way RM ANOVAs found a significant main effect of ethanol exposure for both total $[\mathrm{F}(1,15)=8.988, p=0.0090]$ and maximum $[\mathrm{F}(1,15)=$ $10.8, p=0.0050]$ responses (Fig. $2 \mathrm{f}, \mathrm{g}$ ). A significant interaction between ethanol exposure and time was also found for maximum responses $[\mathrm{F}(2,30)=5.275, p=0.0109]$ with post-hoc analyses revealing a significant reduction at the 12 -h time point $(p \leq 0.001)$. Taken together, these results are consistent with previous observations of a significant decrease in reward sensitivity during acute withdrawal [7] and extend these findings by demonstrating that protracted abstinence is associated with a shift in reward sensitivity in the opposite direction.

RMTg inhibition does not affect reward sensitivity

Given that changes in reward sensitivity during acute withdrawal followed a similar pattern to withdrawal-induced cFos expression in the RMTg, we next examined the possibility that increased activity in this region supports decreased F-R responding. A separate group of rats were tested on the same ICSS procedure following bilateral microinfusion of either saline or muscimol into the RMTg. Figure 3a provides a schematic depiction of the experimental design for these studies. Five rats were excluded prior to CIE or AIR exposure due to failure to acquire ICSS, and two $\mathrm{CIE}$-exposed rats were excluded from final analyses due to misplaced cannula implants resulting in $n=7 /$ group. Figure $3 \mathrm{~b}$ shows the electrode and cannula termination sites of all remaining rats. No between group differences in baseline responding were observed (Fig. S6). Rats were scored for somatic signs of withdrawal immediately prior to each ICSS test session as in the previous experiment (Fig. S7). As shown in Fig. 3d, e, a three-way mixed ANOVA revealed a significant rightward shift in $F-R$ responding in CIE- $[\mathrm{F}(32,38)=3.030, p=0.014]$, but not AIRexposed $[F(32,192)=1.077, p=0.366]$ rats following saline microinfusion, thus replicating our findings from the previous experiment. Surprisingly, temporary inactivation of the RMTg by microinfusion of muscimol had no effect on $F-R$ responding in either group. A significant main effect of ethanol exposure was observed with respect to the total (Fig. $3 f$ ) responses during a session $[F(1,12)=9.233, p=0.0103]$. A similar trend was observed with respect to maximum (Fig. $3 \mathrm{~g}$ ) responses $[\mathrm{F}(1,12)=3.683, p=$ 0.0791]. No significant interactions were observed for either variable indicating that these measures were also unaffected by muscimol microinfusion. These data indicate that although withdrawal-induced alterations in reward sensitivity follow a similar pattern to RMTg cFos expression, inactivation of this region during peak withdrawal is insufficient to normalize this symptom of acute withdrawal.

\section{RMTg inhibition reverses withdrawal-induced anxiety-like} behavior

While unexpected, the fact that the RMTg does not appear to play a role in withdrawal-induced changes in reward sensitivity does not preclude its involvement in regulating other affective symptoms of alcohol withdrawal. Anxiety is a particularly prominent symptom of acute withdrawal that is well-known to promote continued alcohol consumption [39]. We therefore explored the possibility that the RMTg mediates withdrawalinduced increases in anxiety-like behavior. Figure 4a presents a schematic depiction of the experimental design for these studies.
Figure $4 \mathrm{~b}$ shows cannula termination sites for all rats. Rats were scored for somatic signs of withdrawal immediately prior to each anxiety test session as in the previous experiments (Fig. S8). Because anxiogenesis is a robust and reproducible finding during withdrawal from alcohol $[3,9,10]$, we predicted a priori that, compared with saline-treated AIR-exposed rats, saline-treated CIEexposed rats would exhibit a significant increase in anxiety-like behavior during acute withdrawal. This hypothesis was confirmed using light-dark box (Fig. 4c-e), open-field (Fig. 4f-h), and elevated plus maze (Fig. 4i-k) procedures. Compared with saline-treated AIR-exposed rats, saline-treated CIE-exposed rats spent significantly less time in the light side of a light-dark box $[\mathrm{t}(13)=3.934, p=0.0009]$, in the center of an open field [t(16)= $2.194, p=0.0217]$, and in the open arms of an elevated plus maze $[\mathrm{t}(13)=3.889, p=0.0009]$. Analysis of anxiety-like behavior across all four groups revealed that intra-RMTg muscimol prior to testing attenuated withdrawal-induced anxiety-like behavior. Specifically, a two-way RM ANOVA revealed a significant interaction between ethanol exposure and drug treatment for both time $[F(1,28)=$ 5.184, $p=0.0306$ ] and number of entries into the light side $[F(1,28)=8.592, p=0.0067]$ of a light-dark box. Post-hoc tests found a significant difference between saline-treated AIR- and $\mathrm{CIE}$-exposed rats that was not present in muscimol-treated rats (time: $p=0.0019$; entries: $p=0.0003$ ). Muscimol microinfusion also reversed withdrawal-induced decreases in the total crossings in the light-dark box $[F(1,28)=7.561, p=0.0103]$. Significant between factor interactions were also observed when assessing time $[F(1,31)=7.11, p=0.0121]$, the number of entries $[F(1,31)=$ $12.02, p=0.0016]$, and latency to enter the center $[\mathrm{F}(1,31)=4.187$, $p=0.0493$ ] of an open field. Compared with saline-treated AIR-exposed rats, post-hoc tests showed that saline-treated $\mathrm{CIE}$-exposed rats entered the center significantly less frequently $(p=0.0059)$ and trended toward spending less time in the center $(p=0.0626)$, whereas AIR- and CIE-exposed muscimol-treated rats did not differ significantly from each other. In the elevated plus maze, statistical analysis revealed a main effect of ethanol exposure on percent time spent in the open arms $[F(1,26)=$ $6.081, p=0.0206]$, but only a trend toward a significant interaction between ethanol exposure and drug treatment $[F(1,26)=3.465$, $p=0.0740]$. In addition, there was a main effect of drug treatment on percent open-arm entries $[F(1,26)=4.217, p=0.0502]$, but no significant interaction between drug treatment and ethanol exposure. No significant effects of alcohol exposure $[F(1,26)=$ $1.895, p=0.1804]$, drug administration $[F(1,26)=3.022, p=$ $0.0940]$, or interaction between the two factors $[F(1,26)=0.0004$, $p=0.9847$ ] were observed with respect to the number of closedarm entries.

Although the results from the elevated plus maze were less robust than the results obtained with the open-field and light-dark box, a similar trend was observed in all three assays, with absence of withdrawal-induced increase in anxiety-like behavior in CIE-exposed rats that received intra-RMTg muscimol prior to testing. Together, these data suggest that loss of RMTg function attenuates withdrawal-induced anxiety-like behavior. Importantly, the level of intoxication on the morning of testing did not correlate significantly with any measure of anxiety-like behavior in muscimol-infused CIE-exposed rats (data not shown) arguing against the suggestion that higher levels of intoxication prior to withdrawal weakens the effect of intra-RMTg muscimol on performance in this battery of tests.

Inactivation of RMTg has no effect on somatic signs of withdrawal The next set of studies examined the possibility that RMTg inhibition would also attenuate somatic signs of withdrawal. The rationale for this is that somatic signs of withdrawal follow a similar time course to affective symptoms, and performance on anxiety tests could be affected by reversal of somatic withdrawal symptoms. To examine this possibility, we measured the effect of 

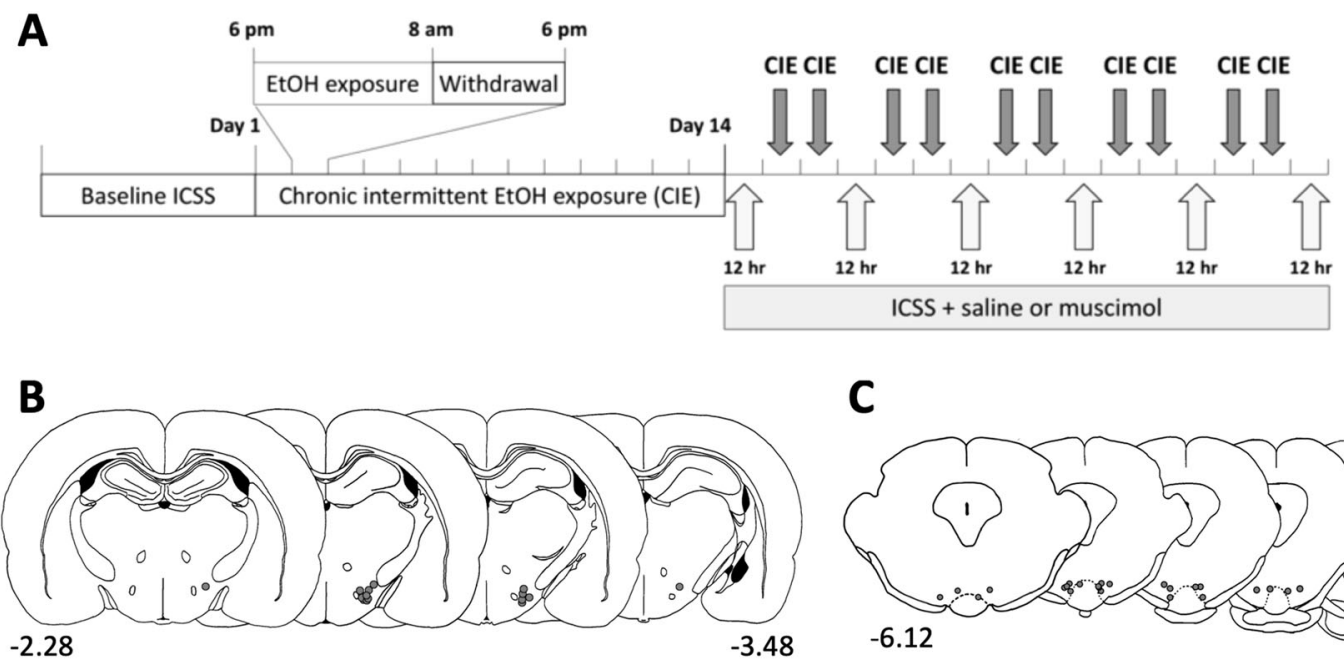

C

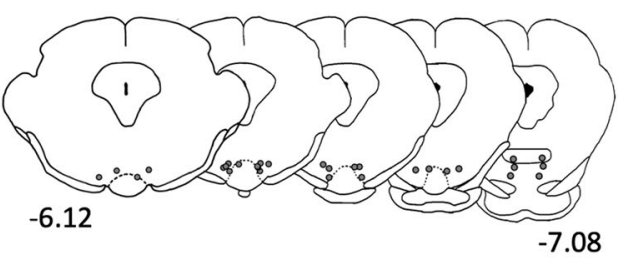

D

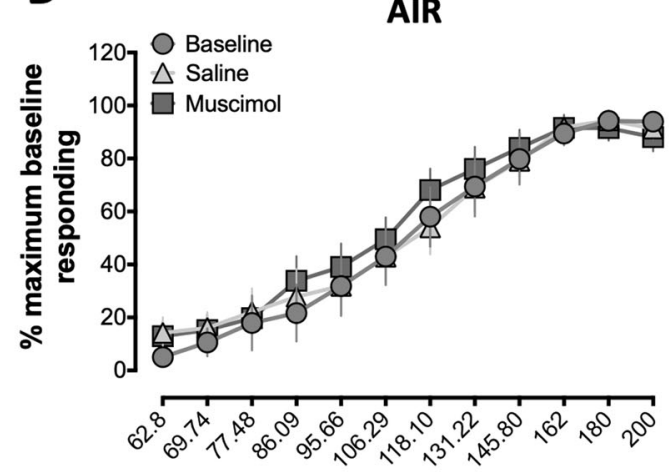

Frequency $(\mathrm{Hz})$

$\mathbf{F}$

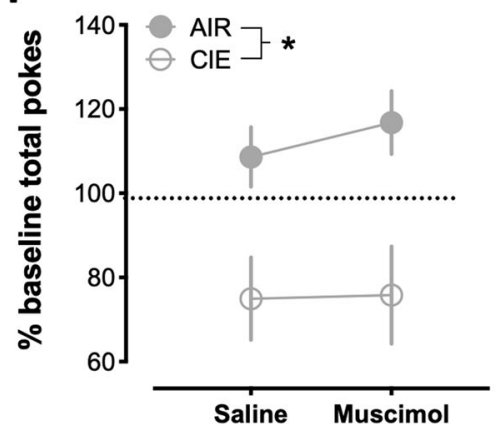

E

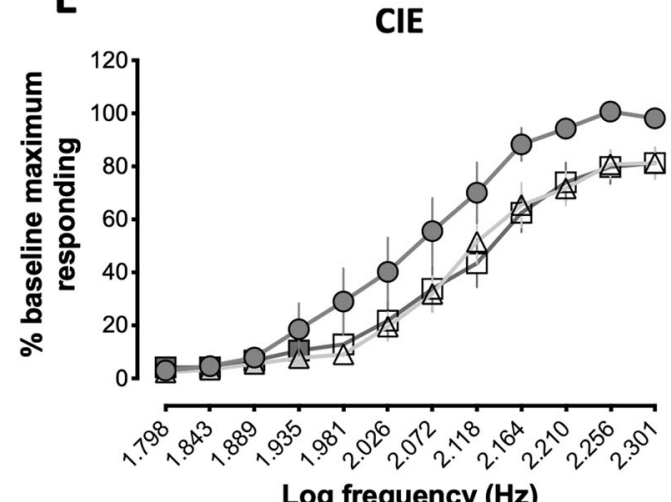

G

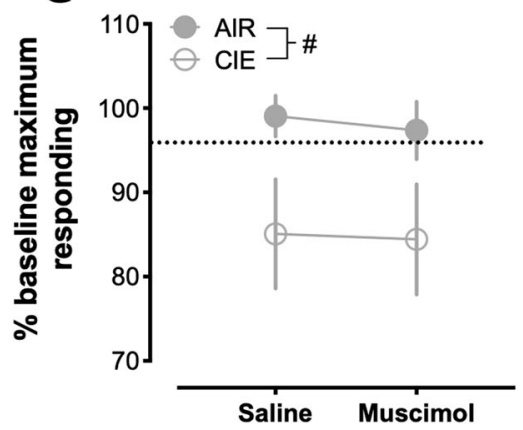

Fig. 3 Effect of RMTg inhibition on withdrawal-induced decrease in reward sensitivity. a Schematic depiction of experimental design, (b) electrode termination, and (c) cannula termination sites. $\mathbf{d}$ F-R responding remained stable regardless of drug administration in AIR-exposed rats. e Inhibition of the RMTg did not alter the withdrawal-induced changes in F-R responding observed in CIE-exposed rats as evidenced by similar responding during trails following microinfusion of either saline or muscimol (open symbols indicate statistical significance from baseline; $p \leq 0.05)$. Microinfusion of muscimol had no effect on withdrawal-induced decreases in the total (f) or maximum ( $\mathbf{g})$ number of responses made during the session. ${ }^{*} p \leq 0.001 ;{ }^{\#} p=0.079 ; \mathrm{AIR}, n=7 ; \mathrm{CIE}, n=7$

intra-RMTg microinfusion of saline or muscimol on somatic signs of withdrawal in $\mathrm{CIE}$-exposed rats $12 \mathrm{~h}$ following removal from ethanol vapor chambers. Intoxication levels achieved during ethanol vapor exposure were similar to that which was observed in the previous experiments (Fig. S9). As shown in Fig. 5, withdrawal scores were not significantly different following microinfusion of either saline or muscimol relative to the preinfusion scores [main effect of drug $\mathrm{F}(1,13)=0.2383, p=0.6336$; main effect of time $F(1,21)=0.1309, p=0.7211$; interaction $F(1,13)=0.1629, p=0.6931]$. This indicates that inhibition of
RMTg activity $12 \mathrm{~h}$ following cessation of ethanol exposure has no effect on somatic signs of withdrawal. Consequently, changes in somatic withdrawal symptoms are unlikely to play a role in the effect of intra-RMTg infusion of muscimol on anxiety-like behavior.

\section{DISCUSSION}

This study revealed that the time course of withdrawal-induced RMTg cFos expression mirrors the severity of somatic and affective withdrawal symptoms. Inhibition of the RMTg $12 \mathrm{~h}$ after cessation 
A

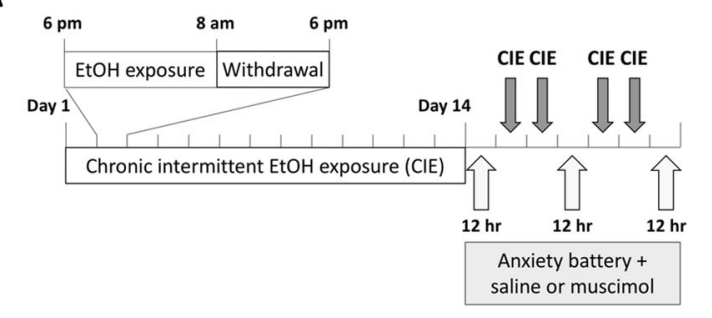

B

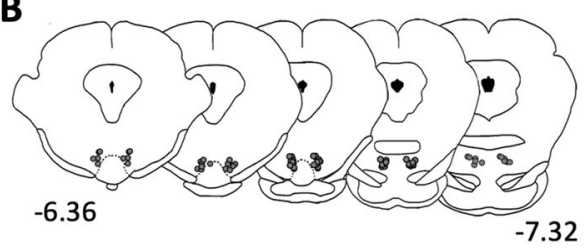

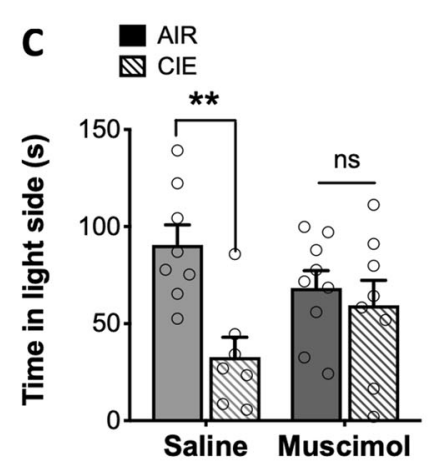

D $\quad$ AIR

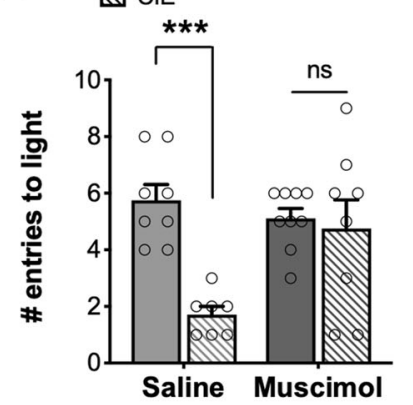

E $\quad$ AIR
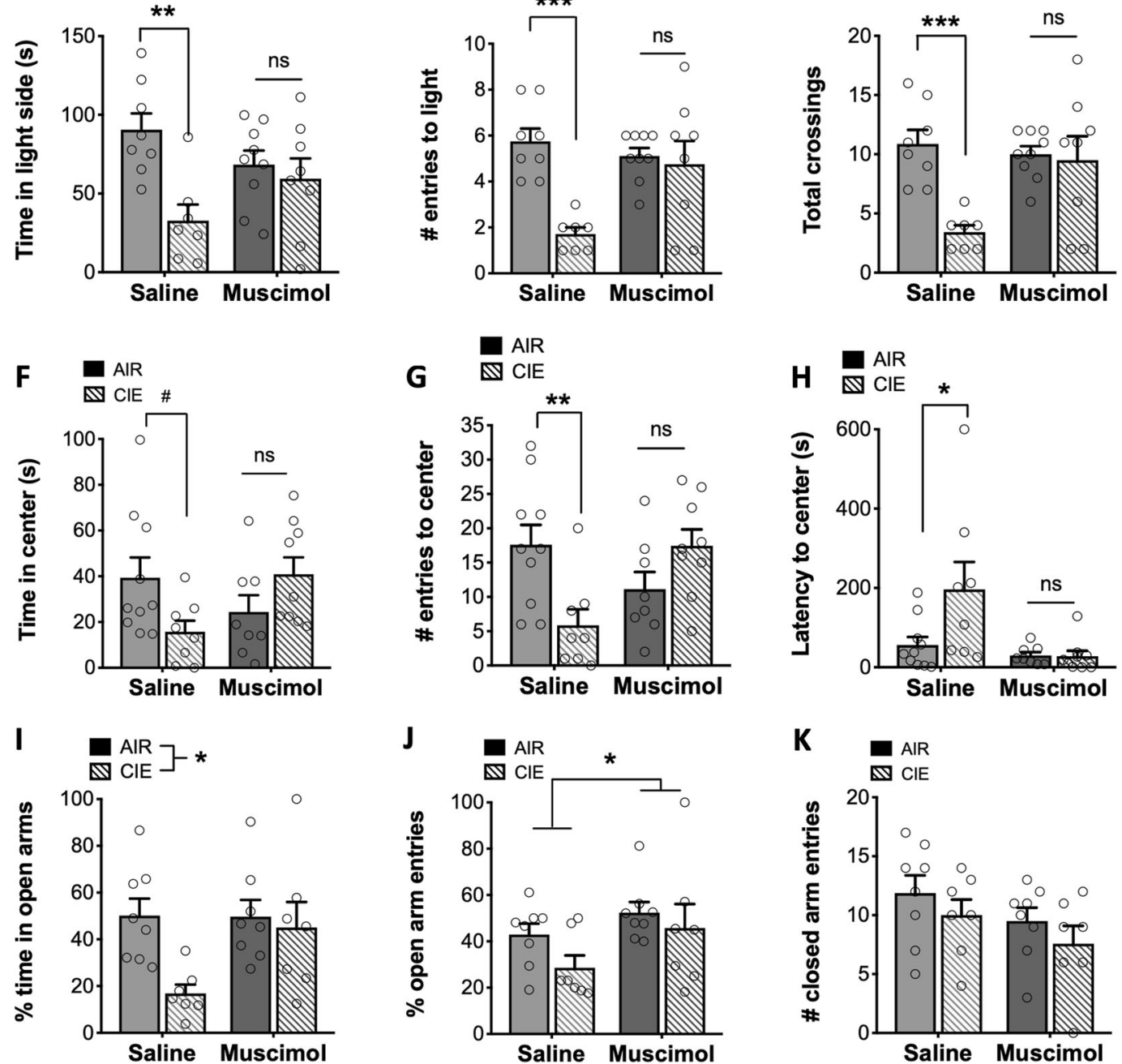

Fig. 4 Effect of RMTg inhibition attenuates withdrawal-induced increase in anxiety-like behavior. a Schematic depiction of experimental design and $\mathbf{b}$ cannula termination sites. CIE-exposed rats that received a microinfusion of saline into the RMTg during withdrawal exhibited a significant increase in anxiety-like behavior compared to AIR controls treated similarly. This effect was attenuated following microinfusion of muscimol into the RMTg during (c-e) light-dark box testing [AIR + saline, $n=8 ;$ CIE + saline, $n=7 ;$ AIR + muscimol, $n=9 ;$ CIE + muscimol, $n=8$ ], (f-h) open field [AIR + saline, $n=10 ; \mathrm{CIE}+$ saline, $n=8 ;$ AIR + muscimol, $n=8 ; \mathrm{CIE}+$ muscimol, $n=9]$, and (i-k) elevated plus maze [AIR + saline, $n=8$; $\mathrm{CIE}+$ saline, $n=7$; AIR + muscimol, $n=8$; CIE + muscimol, $n=7]$. ${ }^{*} p \leq 0.06 ;{ }^{*} p \leq 0.05 ;{ }^{*} p \leq 0.01 ;{ }^{* * *} p \leq 0.001$; ns = not significant

of ethanol exposure, when withdrawal symptoms are at their peak, attenuated heightened anxiety-like behavior, but had no effect on somatic symptoms or withdrawal-induced changes in reward sensitivity. These data suggest that decreased RMTg activity during peak withdrawal attenuates some, but not all, affective symptoms of withdrawal from alcohol.

Acute withdrawal was associated with enhanced cFos expression in the RMTg and $\mathrm{LHb}$ indicating that both regions are hyperactive during this period and may contribute to withdrawal-associated negative affect. In agreement with this, recent work showed that chemogenetic inhibition of the $\mathrm{LHb}$ also decreased withdrawal-induced anxiety-like behavior [40]. Neuronal hyperactivity is a well-known consequence of acute withdrawal [39]. However, not all brain regions exhibit the same time course of cFos expression as evidenced by the absence of withdrawalinduced cFos in the medial habenula, a region that sends a descending projection parallel to the projection from the $\mathrm{LHb}$ to the RMTg. The time course of withdrawal-induced RMTg cFos 


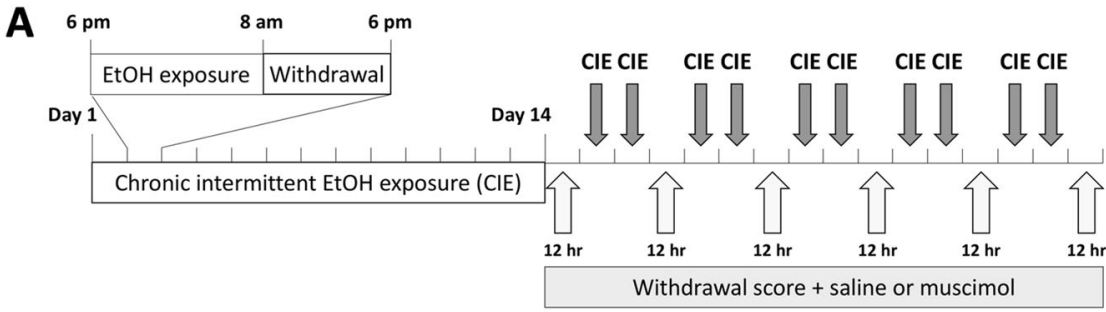

B

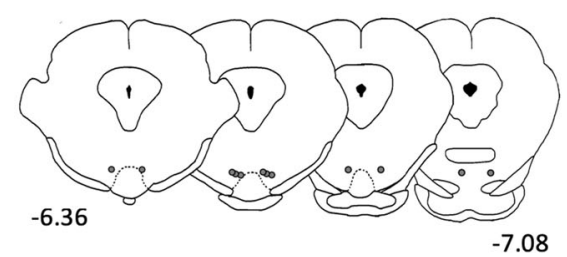

Pre-inf

C 田 Post-inf

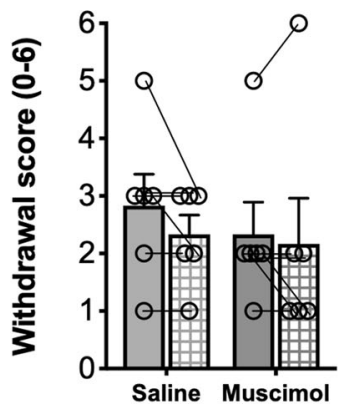

Fig. 5 Effect of RMTg inhibition on somatic signs of withdrawal. a Schematic depiction of experimental design and $\mathbf{b}$ cannula termination sites. c Somatic signs of withdrawal did not differ before and after microinfusion of either saline or muscimol into the RMTg $(n=6)$

expression mirrored previous reports of withdrawal symptom severity [4, 5] leading us to consider the possibility that activity in this region plays a role in mediating symptoms of acute withdrawal.

Using ICSS of the MFB, we observed a significant decrease in reward sensitivity during acute withdrawal. Although this effect was apparent as early as $6 \mathrm{~h}$ into withdrawal, it was the greatest for $12 \mathrm{~h}$ following cessation of ethanol exposure, a time period during which somatic signs of withdrawal were also at their peak. Two previous studies have examined changes in reward sensitivity during acute withdrawal from alcohol $[7,41]$. While findings from these studies are in general agreement with our own, they both employed a current-intensity threshold procedure whereby rats responded to changes in intensity during a single test session. Importantly, changes in intensity alter the anatomical range of neurons activated by electrical current making changes in the response rate to different intensities somewhat difficult to interpret. In contrast, in this study, current was held constant and rats responded to changes in frequency. This approach results in alterations in the excitability of a stable population of neurons. Our findings also expand upon previous work by demonstrating that, in contrast to the decrease in reward sensitivity observed during acute withdrawal, reward sensitivity is significantly increased during protracted abstinence. Overall, these findings agree with a recent meta-analysis suggesting that vulnerability to relapse is mechanistically distinct during different periods of recovery [42].

This study tested the hypothesis that increased RMTg activity plays a role in driving withdrawal-induced alterations in reward sensitivity. This hypothesis was based on our understanding of the inhibitory control that the RMTg exerts over VTA dopamine neurons and the well-characterized decrease in dopamine levels that occurs during acute withdrawal from alcohol. However, the observation that inhibition of the RMTg had no effect on the withdrawal-induced rightward shift in F-R responding argues against this hypothesis. Overlap in the functional connectivity between the RMTg and regions affected by stimulation of the MFB may help to explain these findings. While MFB stimulation increases dopamine release, it does so indirectly by facilitating excitation of VTA dopamine neurons through an intermediate synaptic contact [43]. Midbrain cholinergic nuclei are promising candidates for this effect given that MFB stimulation-induced dopamine release is mediated by activation of cholinergic receptors within the VTA [44]. Notably, the RMTg is reciprocally connected with both the laterodorsal and pedunculopontine tegmental nuclei $[25,26]$. Although speculative, excitatory M3 cholinergic receptors are present within the RMTg [45] leaving open the possibility that MFB stimulation of cholinergic activity could promote RMTg activity potentially minimizing the inhibitory effect of muscimol microinfusion. Alternatively, it is possible that more prolonged inhibition of the RMTg (e.g., during the entire acute withdrawal period) is required to normalize withdrawalinduced changes in reward sensitivity. Indeed, our cFos data suggest that RMTg neurons are hyperactive as early as $6 \mathrm{~h}$ into acute withdrawal. This neuronal hyperactivity may induce lasting neuroadaptations in RMTg function that are not sufficiently reversed by pharmacological inhibition at the $12 \mathrm{~h}$ time point.

In contrast to reward sensitivity, withdrawal-induced anxietylike behavior was significantly attenuated following RMTg inhibition. Although visual inspection of the data indicates that the results from all three anxiety tests are in general agreement, the anxiolytic effect of intra-RMTg muscimol in CIE-exposed rats was more robust in the light-dark box and open-field assays than in the elevated plus maze. CIE-exposed saline-treated rats scored lower on the scale for somatic withdrawal signs prior to elevated plus maze testing than prior to the other tests, suggesting that the lack of statistical significance in this assay could be due to slightly milder withdrawal symptom severity in controls.

It is well known that motor impairments accompany acute withdrawal from alcohol $[39,42]$. Thus, it is possible that the effect of RMTg inhibition on measures of anxiety-like behavior was secondary to its effect on motor performance. Indeed, RMTg inhibition reversed traditionally used measures of locomotor behavior in the light-dark box and open field. However, it had no significant effect on closed-arm entries in the elevated plus maze. In addition, RMTg inhibition did not reverse the withdrawalinduced reduction in maximum responses observed in the ICSS experiment-a measure typically used as an indicator of motor performance independent of changes in reward sensitivity [36]. Furthermore, inhibition of the RMTg did not alter somatic signs of acute withdrawal, which include indices of muscle rigidity and 
impaired gait. Despite the absence of a consistent reversal of motor impairments across tasks, it remains possible that RMTg inhibition altered locomotor behavior in the anxiety tests employed. The tests of anxiety-like behavior used in this study take advantage of the conflict produced by exploratory drive versus risk avoidance. Future work measuring changes in social behavior, startle reflex, grooming, and/or defensive responses may help to discriminate between the effects of RMTg inhibition on different aspects of anxiety-like behavior versus motor performance during withdrawal. However, the validity of each of these assays with respect to measuring anxiety is not without criticism $[46,47]$, and withdrawal-induced changes in motor performance are likely to affect many of these measures as well. Importantly, motor deficits during acute withdrawal may not be independent of affective symptoms, and reversal of this deficit may therefore be equally important in treating symptoms that drive alcohol intake during early abstinence. Additional studies and refinement of existing assays of anxiety-like behavior will be needed to appropriately parse out the contribution that RMTg activity plays in each aspect of performance on these tests.

A number of studies have implicated the RMTg in processing the aversive properties of drugs of abuse including stimulants [26, 30, 31, 48, 49], opiates [50-52], and alcohol [53]. In addition, loss of RMTg function is associated with increased voluntary ethanol intake [54-56], whereas pharmacological activation of the RMTg decreases ethanol preference and consumption [54]. More recently, acute exposure to low-dose ethanol was shown to increase cFos expression in RMTg-projecting LHb neurons [57]. Together, these data suggest that RMTg activity, likely driven by excitatory input from the LHb, suppresses drinking by facilitating signaling of ethanol's aversive properties. Whether RMTg activity plays a similar role during acute withdrawal or if dependenceinduced plasticity alters RMTg function and its role in drinking behavior remains unknown.

Although a major focus of understanding RMTg function is centered on its ability to inhibit midbrain dopamine neurons, it sends projections to other neuromodulatory nuclei, including the laterodorsal and pedunculopontine tegmental nuclei, dorsal raphe, and locus corruleus $[25,26]$. Consequently, withdrawalinduced increases in RMTg activity could have effects on a number of downstream targets, many of which have been functionally implicated in anxiety-like behavior [58-60]. Thus, the exact mechanism by which RMTg activity mediates anxiety-like behavior is unclear and is an important avenue for future research.

In summary, the data presented here demonstrate that heightened activity within the RMTg plays a significant, albeit discrete, role in promoting affective but not somatic symptoms associated with acute withdrawal. Additional work aimed at understanding the mechanism by which the RMTg regulates anxiety-like behavior and the neuroadaptations that occur within this region following chronic ethanol exposure has the potential to uncover new therapeutic targets aimed at preventing relapse to drinking during recovery.

\section{FUNDING AND DISCLOSURE}

The authors thank Nathan Burnham for technical assistance. This work was supported by NIH grants AA024208 (EJG), AA010983 (LJC), AA019967 (LJC), DA037327 (TCJ) and pilot funding from AA010761 (TCJ/EJG). The authors declare no competing interests.

\section{ADDITIONAL INFORMATION}

Supplementary Information accompanies this paper at (https://doi.org/10.1038/ s41386-019-0406-8)

Publisher's note: Springer Nature remains neutral with regard to jurisdictional claims in published maps and institutional affiliations.

\section{REFERENCES}

1. American Psychiatric Association. Substance-related and addictive disorders. In Diagnostic and Statistical Manual of Mental Disorders (DSM-5) American Psychiatric Publishing, p. 481-589, 2013.

2. Hershon HI. Alcohol withdrawal symptoms and drinking behavior. J Stud Alcohol. 1977;38:953-71.

3. Baldwin HA, Rassnick S, Rivier J, Koob GF, Britton KT. CRF antagonist reverses the "anxiogenic" response to ethanol withdrawal in the rat. Psychopharmacol (Berl). 1991;103:227-32.

4. Macey DJ, Schulteis G, Heinrichs SC, Koob GF. Time-dependent quantifiable withdrawal from ethanol in the rat: effect of method of dependence induction. Alcohol Fayettev N. 1996;13:163-70.

5. Rassnick S, Koob GF, Geyer MA. Responding to acoustic startle during chronic ethanol intoxication and withdrawal. Psychopharmacol (Berl). 1992;106:351-8.

6. Roberts AJ, Cole M, Koob GF. Intra-amygdala muscimol decreases operant ethanol self-administration in dependent rats. Alcohol Clin Exp Res. 1996;20:1289-98.

7. Schulteis G, Markou A, Cole M, Koob GF. Decreased brain reward produced by ethanol withdrawal. Proc Natl Acad Sci USA. 1995;92:5880-4.

8. Diana M, Pistis M, Carboni S, Gessa GL, Rossetti ZL. Profound decrement of mesolimbic dopaminergic neuronal activity during ethanol withdrawal syndrome in rats: electrophysiological and biochemical evidence. Proc Natl Acad Sci USA. 1993:90:7966-9.

9. Kliethermes CL. Anxiety-like behaviors following chronic ethanol exposure. Neurosci Biobehav Rev. 2005;28:837-50.

10. Rassnick S, Heinrichs SC, Britton KT, Koob GF. Microinjection of a corticotropinreleasing factor antagonist into the central nucleus of the amygdala reverses anxiogenic-like effects of ethanol withdrawal. Brain Res. 1993;605:25-32.

11. Gilpin NW, Smith AD, Cole M, Weiss F, Koob GF, Richardson HN. Operant behavior and alcohol levels in blood and brain of alcohol-dependent rats. Alcohol Clin Exp Res. 2009;33:2113-23.

12. O'Dell LE, Roberts AJ, Smith RT, Koob GF. Enhanced alcohol self-administration after intermittent versus continuous alcohol vapor exposure. Alcohol Clin Exp Res. 2004;28:1676-82.

13. Roberts AJ, Heyser CJ, Cole M, Griffin P, Koob GF. Excessive ethanol drinking following a history of dependence: animal model of allostasis. Neuropsychopharmacol. Publ Am Coll Neuropsychopharmacol. 2000;22:581-94.

14. Schulteis G, Hyytiä P, Heinrichs SC, Koob GF. Effects of chronic ethanol exposure on oral self-administration of ethanol or saccharin by Wistar rats. Alcohol Clin Exp Res. 1996;20:164-71.

15. Cunningham CL, Fidler TL, Murphy KV, Mulgrew JA, Smitasin PJ. Time-dependent negative reinforcement of ethanol intake by alleviation of acute withdrawal. Biol Psychiatry. 2013;73:249-55.

16. Rossetti ZL, Melis F, Carboni S, Diana M, Gessa GL. Alcohol withdrawal in rats is associated with a marked fall in extraneuronal dopamine. Alcohol Clin Exp Res. 1992;16:529-32.

17. Weiss F, Parsons LH, Schulteis G, Hyytiä $P$, Lorang MT, Bloom FE, et al. Ethanol self-administration restores withdrawal-associated deficiencies in accumbal dopamine and 5-hydroxytryptamine release in dependent rats. J Neurosci J Soc Neurosci. 1996;16:3474-85.

18. Gilpin NW, Herman MA, Roberto M. The central amygdala as an integrative hub for anxiety and alcohol use disorders. Biol Psychiatry. 2015;77:859-69.

19. Funk CK, O'Dell LE, Crawford EF, Koob GF. Corticotropin-releasing factor within the central nucleus of the amygdala mediates enhanced ethanol selfadministration in withdrawn, ethanol-dependent rats. J Neurosci J Soc Neurosci. 2006;26:11324-32.

20. Huang MM, Overstreet DH, Knapp DJ, Angel R, Wills TA, Navarro $M$, et al. Corticotropin-releasing factor (CRF) sensitization of ethanol withdrawal-induced anxiety-like behavior is brain site specific and mediated by CRF-1 receptors: relation to stress-induced sensitization. J Pharmacol Exp Ther. 2010;332:298-307.

21. Kwako LE, Spagnolo PA, Schwandt ML, Thorsell A, George DT, Momenan R, et al. The corticotropin releasing hormone-1 (CRH1) receptor antagonist pexacerfont in alcohol dependence: a randomized controlled experimental medicine study. Neuropsychopharmacol Publ Am Coll Neuropsychopharmacol. 2015;40:1053-63.

22. Schwandt ML, Cortes CR, Kwako LE, George DT, Momenan R, Sinha R, et al. The CRF1 antagonist verucerfont in anxious alcohol-dependent women: translation of neuroendocrine, but not of anti-craving effects. neuropsychopharmacol. Publ Am Coll Neuropsychopharmacol. 2016;41:2818-29.

23. Sanders J, Nemeroff C. The CRF system as a therapeutic target for neuropsychiatric disorders. Trends Pharmacol Sci. 2016;37:1045-54.

24. Pomrenze MB, Fetterly TL, Winder DG, Messing RO. The corticotropin releasing factor receptor 1 in alcohol use disorder: still a valid drug target? Alcohol Clin Exp Res. 2017;41:1986-99.

25. Jhou TC, Geisler S, Marinelli M, Degarmo BA, Zahm DS. The mesopontine rostromedial tegmental nucleus: a structure targeted by the lateral habenula that 
projects to the ventral tegmental area of Tsai and substantia nigra compacta. J Comp Neurol. 2009b;513:566-96.

26. Kaufling J, Veinante P, Pawlowski SA, Freund-Mercier M-J, Barrot M. Afferents to the GABAergic tail of the ventral tegmental area in the rat. J Comp Neurol. 2009;513:597-621.

27. Smith RJ, Vento PJ, Chao YS, Good CH, Jhou TC. Gene expression and neurochemical characterization of the rostromedial tegmental nucleus (RMTg) in rats and mice. Brain Struct Funct. 2019;224:219-38.

28. Yetnikoff L, Cheng A, Lavezzi HN, Parsley KP, Zahm DS. Sources of input to the rostromedial tegmental nucleus, ventral tegmental area and lateral habenula compared: a study in rat. J. Comp. Neurol. 2015; 523:2426-56.

29. Hong S, Jhou TC, Smith M, Saleem KS, Hikosaka O. Negative reward signals from the lateral habenula to dopamine neurons are mediated by rostromedial tegmental nucleus in primates. J Neurosci. 2011;31:11457-71.

30. Jhou TC, Fields HL, Baxter MG, Saper CB, Holland PC. The rostromedial tegmenta nucleus (RMTg), a GABAergic afferent to midbrain dopamine neurons, encodes aversive stimuli and inhibits motor responses. Neuron. 2009a;61:786-800.

31. Jhou TC, Good CH, Rowley C, Xu S, Wang H, Burnham N, et al. Cocaine drives aversive conditioning via delayed activation of dopamine-responsive habenular and midbrain pathways. J Neurosci. 2013;33:7501-12.

32. Vento PJ, Burnham NW, Rowley CS, Jhou TC. Learning from one's mistakes: a dual role for the rostromedial tegmental nucleus in the encoding and expression of punished reward seeking. Biol. Psychiatry. 2017;81:1041-49.

33. National Research Council. Guide for the Care and Use of Laboratory Animals. Eighth Edition. Washington DC: National Academies Press; 2011.

34. Prencipe L, laccheri E, Manzati C. Enzymic ethanol assay: a new colorimetric method based on measurement of hydrogen peroxide. Clin Chem. 1987;33:486-9.

35. Trantham-Davidson H, Burnett EJ, Gass JT, Lopez MF, Mulholland PJ, Centann SW, et al. Chronic alcohol disrupts dopamine receptor activity and the cognitive function of the medial prefrontal cortex. J Neurosci. 2014;34:3706-18.

36. Carlezon WA, Chartoff EH. Intracranial self-stimulation (ICSS) in rodents to study the neurobiology of motivation. Nat Protoc. 2007;2:2987-95.

37. Allen TA, Narayanan NS, Kholodar-Smith DB, Zhao Y, Laubach M, Brown TH Imaging the spread of reversible brain inactivations using fluorescent muscimol. J Neurosci Methods. 2008;171:30-38.

38. Huff ML, LaLumiere RT. The rostromedial tegmental nucleus modulates behavioral inhibition following cocaine self-administration in rats. Neuropsychopharmacol. Publ Am Coll Neuropsychopharmacol. 2015;40:861-73.

39. Heilig M, Egli M, Crabbe JC, Becker HC. Acute withdrawal, protracted abstinence and negative affect in alcoholism: are they linked? Addict Biol. 2010;15:169-84.

40. Kang S, Li J, Zuo W, Fu R, Gregor D, Krnjevic K, et al. Ethanol withdrawal drives anxiety-related behaviors by reducing m-type potassium channel activity in the lateral habenula. neuropsychopharmacol. Publ Am Coll Neuropsychopharmacol. 2017:42:1813-24.

41. Rylkova D, Shah HP, Small E, Bruijnzeel AW. Deficit in brain reward function and acute and protracted anxiety-like behavior after discontinuation of a chronic alcohol liquid diet in rats. Psychopharmacol (Berl). 2009;203:629-40.

42. Hirth N, Meinhardt MW, Noori HR, Salgado H, Torres-Ramirez O, Uhrig S, et al. Convergent evidence from alcohol-dependent humans and rats for a hyperdopaminergic state in protracted abstinence. Proc Natl Acad Sci USA. 2016;113:3024-9.

43. You ZB, Chen YQ, Wise RA. Dopamine and glutamate release in the nucleus accumbens and ventral tegmental area of rat following lateral hypothalamic selfstimulation. Neuroscience. 2001;107:629-39.
44. Kofman O, McGlynn SM, Olmstead MC, Yeomans JS. Differential effects of atropine, procaine and dopamine in the rat ventral tegmentum on lateral hypothalamic rewarding brain stimulation. Behav Brain Res. 1990;38:55-68.

45. Steidl S, Wasserman DI, Blaha CD, Yeomans JS. Opioid-induced rewards, locomotion, and dopamine activation: A proposed model for control by mesopontine and rostromedial tegmental neurons. Neurosci Biobehav Rev. 2017;83:72-82.

46. Steimer T. Animal models of anxiety disorders in rats and mice: some conceptual issues. Dialog- Clin Neurosci. 2011;13:495-506.

47. Ennaceur A. Tests of unconditioned anxiety-pitfalls and disappointments. Physiol Behav. 2014;135:55-71.

48. Kaufling J, Waltisperger E, Bourdy R, Valera A, Veinante $P$, Freund-Mercier $M-J$ et al. Pharmacological recruitment of the GABAergic tail of the ventral tegmental area by acute drug exposure. Br J Pharmacol. 2010;161:1677-91.

49. Zahm DS, Becker ML, Freiman AJ, Strauch S, Degarmo B, Geisler S, et al. Fos after single and repeated self-administration of cocaine and saline in the rat: emphasis on the Basal forebrain and recalibration of expression. Neuropsychopharmacol Publ Am Coll Neuropsychopharmacol. 2010;35:445-63.

50. Lecca S, Melis M, Luchicchi A, Ennas MG, Castelli MP, Muntoni AL, et al. Effects of drugs of abuse on putative rostromedial tegmental neurons, inhibitory afferents to midbrain dopamine cells. Neuropsychopharmacol Publ Am Coll Neuropsychopharmacol. 2011;36:589-602.

51. Lecca $S$, Melis $M$, Luchicchi A, Muntoni AL, Pistis M. Inhibitory inputs from rostromedial tegmental neurons regulate spontaneous activity of midbrain dopamine cells and their responses to drugs of abuse. Neuropsychopharmacol Publ Am Coll Neuropsychopharmacol. 2012;37:1164-76.

52. Matsui A, Williams JT. Opioid-sensitive GABA inputs from rostromedial tegmental nucleus synapse onto midbrain dopamine neurons. J Neurosci J Soc Neurosci. 2011;31:17729-35.

53. Glover EJ, McDougle MJ, Siegel GS, Jhou TC, Chandler LJ. Role for the ros tromedial tegmental nucleus in signaling the aversive properties of alcohol. Alcohol Clin Exp Res. 2016;40:1651-61.

54. Fu R, Zuo W, Gregor D, Li J, Grech D, Ye J-H. Pharmacological manipulation of the rostromedial tegmental nucleus changes voluntary and operant ethanol selfadministration in rats. Alcohol Clin Exp Res. 2016b;40:572-82.

55. Sheth C, Furlong TM, Keefe KA, Taha SA. Lesion of the rostromedial tegmental nucleus increases voluntary ethanol consumption and accelerates extinction of ethanol-induced conditioned taste aversion. Psychopharmacol (Berl). 2016; 233:3737-49.

56. Fu R, Chen X, Zuo W, Li J, Kang S, Zhou L-H, et al. Ablation of $\mu$ opioid receptorexpressing GABA neurons in rostromedial tegmental nucleus increases ethanol consumption and regulates ethanol-related behaviors. Neuropharmacology. 2016a;107:58-67.

57. Fu R, Mei Q, Zuo W, Li J, Gregor D, Bekker A, et al. Low-dose ethanol excites lateral habenula neurons projecting to VTA, RMTg, and raphe. Int J Physiol Pathophysiol Pharmacol. 2017;9:217-30.

58. Montoya A, Bruins R, Katzman MA, Blier P. The noradrenergic paradox: implications in the management of depression and anxiety. Neuropsychiatr Dis Treat. 2016;12:541-57.

59. Picciotto MR, Lewis AS, van Schalkwyk Gl, Mineur YS. Mood and anxiety regulation by nicotinic acetylcholine receptors: a potential pathway to modulate aggression and related behavioral states. Neuropharmacology. 2015;96:235-43.

60. Żmudzka E, Sałaciak K, Sapa J, Pytka K. Serotonin receptors in depression and anxiety: insights from animal studies. Life Sci. 2018;210:106-24. 